\title{
ON EXTREME POINTS OF SUBORDINATION FAMILIES
}

\author{
YUSUF ABU MUHANNA
}

ABSTRACT. Let $F$ be the set of analytic functions in $U=\{z:|z|<1\}$ subordinate to a univalent function $f$. Let $D=f(U)$. For $g(z)=f(\phi(z)) \in F$, let $\lambda(\theta)$ denote the distance between $g\left(e^{\prime \theta}\right)$ and $\partial D$ (boundary of $D$ ). We obtain the following results.

(1) If $f^{\prime}$ is Nevanlinna then $\int_{0}^{2 \pi} \log \lambda(\theta) d \theta=-\infty$ if and only if

$$
\int_{0}^{2 \pi} \log \left(1-\left|\phi\left(e^{i \theta}\right)\right|\right) d \theta=-\infty .
$$

(2) If $g$ is an extreme point of the closed convex hull of $F$ then

$$
\int_{0}^{2 \pi} \log \left(1-\left|\phi\left(e^{i \theta}\right)\right|\right) d \theta=-\infty
$$

for the case when $D$ is a Jordan domain subset to a half-plane and $f^{\prime}$ is Nevanlinna.

1. Introduction. Let $U=\{z:|z|<1\}$ and let $A$ denote the set of functions analytic in $U$. Let $B_{0}$ denote the subset of $A$ consisting of functions $\phi$ that satisfy $|\phi(z)|<1$ for $z \in U$ and $\phi(0)=0$.

Throughout this paper we assume that $f \in A$ and $f$ is univalent in $U$. Let $F$ denote the subset of $A$ consisting of functions $g$ that are subordinate to $f$ in $U$. This means that $g \in A, g(0)=f(0)$ and $g(U) \subset f(U)$. These conditions are equivalent to the existence of $\phi \in B_{0}$ so that $g(z)=f(\phi(z))$. $F$ is characterized by

$$
g(z)=f(\phi(z))
$$

where $\phi \in B_{0}$. Equation (1) defines a one-to-one correspondence between $F$ and $B_{0}$.

Let $D$ denote $f(U)$. For $g \in F$, let

$$
g\left(e^{i \theta}\right)=\lim _{r \rightarrow 1} g\left(r e^{i \theta}\right) .
$$

Since $f \in H^{p}$, for $p<\frac{1}{2}, g\left(e^{i \theta}\right)$ exists almost everywhere. Let $\lambda(\theta)$ denote the distance between $g\left(e^{i \theta}\right)$ and $\partial D$ where $\partial D$ denotes the boundary of $D$. T. $H$. MacGregor and the author [1] proved that if $f$ is convex, bounded, and if $\partial D$ is sufficiently smooth, then $g$ is an extreme point of $F$ if and only if

$$
\int_{0}^{2 \pi} \log \lambda(\theta) d \theta=-\infty .
$$

This result implies the well-known fact that $\phi$ is an extreme point of $B_{0}$ if and only if

$$
\int_{0}^{2 \pi} \log \left(1-\left|\phi\left(e^{i \theta}\right)\right|\right) d \theta=-\infty
$$

Received by the editors May 13, 1982. Presented at the AMS 795th meeting in Bellingham, Washington, June 19, 1982.

1980 Mathematics Subject Classification. Primary 30C80; Secondary 30C55.

Key words and phrases. Analytic function, bounded function, convex function, extreme point, Jordan domain, Nevanlinna class, subordination, univalent function. 
[2, p. 125]. Other results in this direction can be found in [1, 4,5 and 7].

It was also proved [1] that when $f$ is bounded, convex and $\partial D$ is sufficiently smooth the correspondence between $F$ and $B_{0}$ given by $g(z)=f(\phi(z))$ provides a one-to-one correspondence between the extreme points of $B_{0}$ and the extreme points of $F$. This is to say,

$$
\int_{0}^{2 \pi} \log \lambda(\theta) d \theta=-\infty \text { if and only if } \int_{0}^{2 \pi} \log \left(1-\left|\phi\left(e^{i \theta}\right)\right|\right) d \theta=-\infty
$$

In $\$ 2$, we prove that statement (5) holds for the case when $f$ is univalent and $f^{\prime}$ is in the Nevanlinna class of analytic functions.

In $\S 3$, we give a necessary condition on the extreme points of the closed convex hull of $F$ for the case when $D=f(U)$ lies in a half-plane and $\partial D$ is a Jordan curve.

2. Functions subordinate to a univalent function with a Nevanlinna derivative. We let $d(z, \Gamma)$ denote the distance between $z$ and $a$ closed set $\Gamma, m(A)$ denote the Lebesgue measure of $A$ and $\log ^{+} x=\max \{0, \log x\}$.

THEOREM 1. Let $f$ be analytic and univalent in $U$. Assume that $f^{\prime}$ is in the Nevanlinna class. Let $D$ denote $f(U)$ and let $F$ denote the set of functions subordinate to $f$. For $g(z)=f(\phi(z)) \in F$, let $\lambda(\theta)$ denote $d\left(g\left(e^{i \theta}\right), \partial D\right)$. Then:

(a) $\int_{0}^{2 \pi} \log ^{+} \lambda(\theta) d \theta$ is convergent.

(b) $\int_{0}^{2 \pi} \log \lambda(\theta) d \theta=-\infty$ if and only if $\int_{0}^{2 \pi} \log \left(1-\left|\phi\left(e^{i \theta}\right)\right|\right) d \theta=-\infty$.

Proof. Since $f$ is univalent, it follows that

$$
\frac{1}{4}\left(1-|z|^{2}\right)\left|f^{\prime}(z)\right| \leqslant d(f(z), \partial D) \leqslant\left(1-|z|^{2}\right)\left|f^{\prime}(z)\right|, \quad z \in U
$$

[8, p. 22]. When $g\left(e^{i \theta}\right)$ and $\phi\left(e^{i \theta}\right)$ exist and $\left|\phi\left(e^{i \theta}\right)\right|<1$, we obtain

$$
\text { (7) } \frac{1}{4}\left(1-\left|\phi\left(e^{i \theta}\right)\right|^{2}\right)\left|f^{\prime}\left(\phi\left(e^{i \theta}\right)\right)\right| \leqslant \lambda(\theta) \leqslant\left(1-\left|\phi\left(e^{i \theta}\right)\right|^{2}\right)\left|f^{\prime}\left(\phi\left(e^{i \theta}\right)\right)\right| \text {. }
$$

Hence (7) implies that $\lambda(\theta) \leqslant\left|f^{\prime}\left(\phi\left(e^{i \theta}\right)\right)\right|$ and consequently $0 \leqslant \log ^{+} \lambda(\theta) \leqslant$ $\log ^{+}\left|f^{\prime}\left(\phi\left(e^{i \theta}\right)\right)\right|$. Since $f^{\prime}$ is Nevanlinna and $\phi$ is bounded, it follows that $f^{\prime}(\phi(z))$ is also Nevanlinna. Hence $\log \left|f^{\prime}\left(\phi\left(e^{i \theta}\right)\right)\right| \in L^{1}$ and in particular $\log ^{+}\left|f^{\prime}\left(\phi\left(e^{i \theta}\right)\right)\right| \in L^{1}$ [2, p. 16]. Therefore, $\int_{0}^{2 \pi} \log ^{+} \lambda(\theta) d \theta$ is convergent, which is part (a).

Next, let $A=\left\{\theta: g\left(e^{i \theta}\right)\right.$ exists and $\left.\lambda(\theta)=0\right\}$. If $m(A)>0$ then it follows that $\int_{0}^{2 \pi} \log \lambda(\theta) d \theta=\int_{0}^{2 \pi} \log \left(1-\left|\phi\left(e^{i \theta}\right)\right|\right) d \theta=-\infty$. Assume for the rest of the proof that $m(A)=0$. This implies that (7) holds for almost all $\theta$. The fact that $1 \leqslant 1+$ $\left|\phi\left(e^{i \theta}\right)\right| \leqslant 2$ reduces $(7)$ to

$$
\frac{1}{4}\left(1-\left|\phi\left(e^{i \theta}\right)\right|\right) f^{\prime}\left(\phi\left(e^{i \theta}\right)\right)\left|\leqslant \lambda(\theta) \leqslant 2\left(1-\left|\phi\left(e^{i \theta}\right)\right|\right)\right| f^{\prime}\left(\phi\left(e^{i \theta}\right)\right) \mid
$$

which also holds for almost every $\theta$. Thus we conclude that $-\infty \leqslant \int_{0}^{2 \pi} \log \lambda(\theta) d \theta<$ $M$, for some constant $M$, because $\log ^{+} \lambda(\theta) \in L^{1}$. This together with (8) implies that (9)

$$
\begin{aligned}
&-2 \pi \log 4+\int_{0}^{2 \pi} \log \left|f^{\prime}\left(\phi\left(e^{i \theta}\right)\right)\right| d \theta+\int_{0}^{2 \pi} \log \left(1-\left|\phi\left(e^{i \theta}\right)\right|\right) d \theta \leqslant \int_{0}^{2 \pi} \log \lambda(\theta) d \theta \\
& \leqslant \int_{0}^{2 \pi} \log \left(1-\left|\phi\left(e^{i \theta}\right)\right|\right) d \theta+\int_{0}^{2 \pi} \log \left|f^{\prime}\left(\phi\left(e^{i \theta}\right)\right)\right| d \theta+2 \pi \log 2
\end{aligned}
$$

and so part (b) follows. 
We close this section by noting that the conclusion of Theorem 1 is true for the case when $g$ is subordinate to a close to convex function $f$. This is so because it was shown that $f^{\prime} \in H^{1 / 3}[3]$ and thus $f^{\prime}$ is Nevanlinna.

\section{Jordan domains. We let $c A$ denote $\mathrm{C} \backslash A$.}

LEMMA 1. Let $D$ be a bounded Jordan domain ( $\partial D$ is a Jordan curve). Let $g$ be a nonconstant bounded analytic function in $U$. If $g\left(e^{i \theta}\right) \in \bar{D}$ for almost all $\theta$ then $g(U) \subset D$.

Proof. Let $G=g(U)$. We want to show that $G \subset D$. We shall show first that $\partial \bar{G} \subset D$. Assume, to the contrary, that there is a point $w_{0} \in \partial \bar{G}$ and $w_{0} \notin \bar{D}$. Let $\varepsilon=d\left(w_{0}, D\right)$. Since $w_{0} \in \partial \bar{G}$ and $w_{0} \notin \bar{D}$, there exists a point $w_{1} \in c \bar{G}$ and $w_{1} \notin \bar{D}$ so that $\left|w_{0}-w_{1}\right|<\varepsilon / 2$. It follows that $d\left(w_{1}, D\right)>\varepsilon / 2$. Let

$$
h(z)=\frac{1}{g(z)-w_{1}}, \quad z \in U,
$$

$h(z)$ is analytic, bounded and $h\left(e^{i \theta}\right)=1 /\left(g\left(e^{i \theta}\right)-w_{1}\right)$ for almost all $\theta$. Since $g\left(e^{i \theta}\right) \in \bar{D}$ for almost all $\theta$, it follows that $\left|h\left(e^{i \theta}\right)\right| \leqslant 2 / \varepsilon$ for almost all $\theta$. The Poisson Formula implies that $|h(z)| \leqslant 2 / \varepsilon$ for every $z \in U$. This contradicts $\left|w_{1}-w_{0}\right|<\varepsilon / 2$. Hence $\partial \bar{G} \in \bar{D}$.

Next, we shall show that $L=\bar{G} \cap c \bar{D}$ is open. Let $w \in L$. Since $c \bar{D}$ is open, there is a neighborhood of $w, N_{\varepsilon}(w)$, so that $N_{\varepsilon}(w) \subset c \bar{D} \cdot N_{\varepsilon / 2}(w) \subset L$, because if not then $N_{\varepsilon / 2}(w) \cap c \bar{G} \neq \varnothing$ and, since $N_{\varepsilon / 2}(w) \cap \bar{G} \neq \varnothing$, one concludes that $N_{\varepsilon / 2}(w) \cap \partial \bar{G}$ $\neq \varnothing$. Thus there exists $w_{0} \in N_{\varepsilon / 2}(w) \cap \partial \bar{G}$ and $w_{0} \notin \bar{D}$. This then contradicts the first part of the proof of the lemma. Hence $L$ is open.

Let $u \in \partial L$ and assume that $u \notin \bar{D}$. Since $c L=c \bar{G} \cup \bar{D}$ it follows that every neighborhood of $u$, with radius less than $d(u, D)$, intersects $c \bar{G}$. This implies that $u \in \partial \bar{D}$ and consequently $u \in \bar{D}$. Hence $\partial L \subset \partial D$ and consequently $c \bar{D}=L \cup(c \bar{L}$ $\cap c \bar{D}$ ). Since $c \bar{D}$ is connected ( $\partial D$ is a Jordan curve) and since $L$ is bounded, we conclude that $L$ is empty and then $\bar{G} \subset \bar{D}$. This and Jordan's Theorem [7,p. 115] imply that $G \subset D$.

The following lemma is a generalization of Lemma 1.

LEMMA 2. Let $D$ be a Jordan domain subset to a half-plane $H$. Let $g$ be a nonconstant function analytic in $U$ so that $g(U) \subset H$. If $g\left(e^{i \theta}\right) \in \bar{D}$ for almost every $\theta$ then $g(U) \subset D$.

Proof. Let $T$ be a möbius transformation that maps $H$ onto $U$. Let $h(z)=T(g(z))$. $h(z)$ is bounded, analytic, $h\left(e^{i \theta}\right)=T\left(g\left(e^{i \theta}\right)\right)$ exists for almost all $\theta$ and $h\left(e^{i \theta}\right)$ $\in \overline{T(D)}$. Since $T$ is a homeomorphism and $\partial D$ is a Jordan curve, it follows that $\partial(T(D))$ is a Jordan curve. Hence, by Lemma $1, h(U) \subset T(D)$ and consequently $g(U) \subset D$.

We now apply Lemma 2 to get the following theorem.

THEOREM 2. Let $f$ be a univalent analytic function in $U$. Assume that $D=f(U)$ is a Jordan domain subset to a half-plane $H$. Let $F$ be the set of analytic functions subordinate to $f$. If $g$ is an extreme point of the closed convex hull of $F$ then $\int_{0}^{2 \pi} \log \lambda(\theta) /(1+\lambda(\theta)) d \theta=-\infty$. 
REMARK. $\lambda(\theta) /(1+\lambda(\theta))$ can be replaced by $\lambda(\theta)$ when $f$ is bounded.

Proof. Assume that $\int_{0}^{2 \pi} \log [\lambda(\theta) /(1+\lambda(\theta))] d \theta>-\infty$. Since $\lambda(\theta) /(1+\lambda(\theta))$ $<1, \log [\lambda(\theta) /(1+\lambda(\theta))] \in L^{1}$. Let

$$
h(z)=z \exp \left\{\frac{1}{2 \pi} \int_{0}^{2 \pi} \frac{e^{i t}+z}{e^{i t}-z} \log \frac{\lambda(t)}{1+\lambda(t)} d t\right\} .
$$

It is known that $h \in H^{\infty}$ and $\left|h\left(e^{i \theta}\right)\right|=\lambda(\theta) /(1+\lambda(\theta))$ for almost all $\theta$ [2, pp. 24, 126]. Since $\left|h\left(e^{i \theta}\right)\right| \leqslant \lambda(\theta)$, it follows that $g\left(e^{i \theta}\right) \pm \mathrm{h}\left(e^{i \theta}\right) \in \bar{D}$ for almost all $\theta$. Moreover, $h \in H^{\infty}$ implies that $g(z) \pm h(z)$ is in a half-plane $H_{1}$, containing $H$, for all $z \in U$. Thus, by Lemma 2, it follows that $g(z) \pm h(z) \in D$ for all $z \in U$ and so $g(z) \pm h(z) \in F$. Since $h \neq 0, g$ cannot be an extreme point.

We come now to the main result of this section.

THEOREM 3. Let $f$ be a univalent analytic function in $U$. Assume that $f^{\prime}$ is Nevanlinna and $D=f(U)$ is a Jordan domain subset to a half-plane $H$. Let $F$ be the set of analytic functions subordinate to $f$. If $g(z)=f(\phi(z))$ is an extreme point of the closed convex hull of $F$ then $\int_{0}^{2 \pi} \log \left(1-\left|\phi\left(e^{i \theta}\right)\right|\right) d \theta=-\infty$.

REMARK. In other words, $\{g \in F: g$ is an extreme point of the closed convex hull of $F\} \subset\left\{f(\phi): \phi\right.$ is an extreme point of $\left.B_{0}\right\}$.

Proof. Theorem 2 implies that $\int_{0}^{2 \pi} \log [\lambda(\theta) /(1+\lambda(\theta))] d \theta=-\infty$. Let $E=\{\theta$ : $\lambda(\theta)$ exists and $\lambda(\theta) \leqslant 1\}$ and let $G=\{\theta: \lambda(\theta)$ exists and $\lambda(\theta)>1\} \cdot m(E \cup G)=$ $2 \pi$. For $\theta \in E$, we have

$$
\frac{\lambda(\theta)}{2} \leqslant \frac{\lambda(\theta)}{1+\lambda(\theta)} \leqslant \lambda(\theta)
$$

and for $\theta \in G$, we have $1+\lambda(\theta)<2 \lambda(\theta)$ and so

$$
\frac{1}{2}<\frac{\lambda(\theta)}{1+\lambda(\theta)}<1 .
$$

(13) implies that $\int_{G} \log [\lambda(\theta) /(1+\lambda(\theta))] d \theta$ is convergent. Therefore,

$$
\int_{0}^{2 \pi} \log \frac{\lambda(\theta)}{1+\lambda(\theta)} d \theta=\int_{E} \log \frac{\lambda(\theta)}{1+\lambda(\theta)} d \theta=-\infty
$$

and by (12) $\int_{E} \log \lambda(\theta) d \theta=-\infty$. Because of Theorem 1 this gives that $\int_{0}^{2 \pi} \log \lambda(\theta) d \theta=-\infty$ and consequently $\int_{0}^{2 \pi} \log \left(1-\left|\phi\left(e^{i \theta}\right)\right|\right) d \theta=-\infty$.

REMARKS. 1. The conclusion of Theorem 3 follows for the case when $f$ is convex. This is because $f(D)$ is a Jordan domain and $f^{\prime} \in H^{1 / 2}[3]$.

2. Theorem 2 was proved by T. H. MacGregor and the author [1] for the case when $f$ is convex and $f(U)$ is not a half-plane.

3. The converse of Theorem 3 does not hold in general. For example, the extreme points of $F$, when $f=(1+z) /(1-z)$, are characterized by

$$
g=\frac{1+x z}{1-x z}, \quad|x|=1 .
$$

Other examples in [1 and 6] show this claim.

4. We conjecture that Theorems 1 and 3 hold for any unrestricted univalent function $f$. 
ACKnOWLedgement. The author wishes to thank the University of Petroleum and Minerals for supporting this research.

\section{REFERENCES}

1. Y. Abu Muhanna and T. H. MacGregor, Extreme points of families of analytic functions subordinate to convex mappings, Math. Z. 176 (1981), 511-519.

2. P. L. Duren. Theory of $H^{p}$ spaces, Academic Press, New York, 1970.

3. P. J. Eenigenburg and F. R. Keogh, The Hardy class of some univalent functions and their derivatives, Michigan Math. J. 17 (1970), 335-346.

4. D. J. Hallenbeck and T. H. MacGregor, Subordination and extreme point theon, Pacific J. Math. 50 (1974), 455-468.

5. H. M. Hilden, $A$ characterization of the extreme points of some convex sets of analytic functions, Duke Math. J. 37 (1970), 715-723.

6. J. G. Milcetich. On the extreme points of some sets of analytic functions, Proc. Amer. Math. Soc. 45 (1974), 223-228.

7. M. H. Neuman, Elements of the topologr of plane sets of points, Cambridge Univ. Press, Cambridge. 1964.

8. Chr. Pommerenke, Univalent functions, Vandenhoeck \& Ruprecht, Göttingen, 1975.

Department of Mathematical Sciences, University of Petroleum and Minerals, Dhahran, SAUdi ARABIA 